\title{
Forskolin promotes vasculogenic mimicry and invasion via Notch-1-activated epithelial-to-mesenchymal transition in syncytiolization of trophoblast cells in choriocarcinoma
}

\author{
YAN XUE $^{1 *}$, RONG SUN ${ }^{1,2^{*}}$, WEI ZHENG ${ }^{1,3}$, LEI YANG $^{1}$ and RUIFANG AN ${ }^{1}$ \\ ${ }^{1}$ Department of Gynecology and Obstetrics, The First Affiliated Hospital of Xi'an Jiaotong University, \\ Xi'an, Shaanxi 710061; ${ }^{2}$ Department of Gynecology and Obstetrics, Obstetrics and Gynecology Hospital of \\ Fudan University, Shanghai 200011; ${ }^{3}$ Department of Gynecology and Obstetrics, \\ Ningbo Medical Center Lihuili Eastern Hospital, Ningbo, Zhejiang 315040, P.R. China
}

Received July 1, 2019; Accepted January 23, 2020

DOI: 10.3892/ijo.2020.4997

\begin{abstract}
Choriocarcinoma (CC) is characterized by earlier blood metastasis compared with other female genital tumors and a high incidence of massive hemorrhage. Vasculogenic mimicry (VM) is highly associated with metastasis, and syncytiotrophoblast is involved in the formation of VM in CC. Forskolin is a typical activator of the cAMP pathway, which is involved in the syncytiolization of trophoblastic cells. In the present study, to determine the effects and mechanism of forskolin on cell invasion and VM during syncytiolization in vitro and in vivo, JEG-3 and JAR cell lines were treated with $100 \mu \mathrm{M}$ forskolin for $48 \mathrm{~h}$, and wound healing and invasion assays were used to verify cell migratory and invasive capacities. A 3D culture and tube formation assays were established to detect VM. Variation of morphology and markers of the epithelial-to-mesenchymal transition (EMT) were assessed, and the role of the Notch signaling pathway was investigated in CC cells treated with forskolin. The results of the present study demonstrated that $100 \mu \mathrm{M}$ forskolin induced syncytiolization of trophoblastic cells and enhanced the migratory and invasive abilities of JEG-3 and JAR cell lines. In addition, the capacity of VM was significantly increased, whereas tube formation ability was decreased by forskolin in vitro and in vivo compared with the respective control groups. The cellular morphology exhibited EMT during the
\end{abstract}

Correspondence to: Dr Ruifang An or Dr Yan Xue, Department of Gynecology and Obstetrics, The First Affiliated Hospital of Xi'an Jiaotong University, 277 Western YanTa Road, Xi'an, Shaanxi 710061, P.R. China

E-mail: ruifangAn@126.com

E-mail: snowcathy@xjtu.edu.cn

${ }^{*}$ Contributed equally

Key words: forskolin, vasculogenic mimicry, epithelial-tomesenchymal transition, Notch, choriocarcinoma syncytiolization process, which was further supported by the changes in EMT marker expression, including downregulation of E-cadherin and cytokeratin and upregulation of N-cadherin, vimentin and zinc finger E-box-binding homeobox 1 . The Notch-1 signaling pathway was activated to induce EMT in forskolin-induced VM process in CC cells, and VM and EMT could be reversed by using the $\gamma$-secretase inhibitor DAPT to block the Notch-1 pathway. Overall, the results of the present study demonstrated that forskolin enhanced the capacity of VM formation and metastasis through Notch-1-activated EMT in the syncytiolization of trophoblastic cells.

\section{Introduction}

Choriocarcinoma (CC) is a pregnancy-associated tumor characterized by massive hemorrhage and earlier hematogenous metastasis compared with other female genital tumors (1). According to National Comprehensive Cancer Network guidelines, with the development of chemotherapy, the overall cure rate of patients with high-risk CC has reached $\sim 90 \%$ worldwide (2). However, multi-drug resistant CC still remains to be a challenge in the clinical setting (3). Angiogenetic ability is closely associated with the malignancy and chemo-sensitivity of tumors (4). Vasculogenic mimicry (VM) is the process by which aggressive cancer cells can form highly patterned vascular channels without the presence of endothelial cells (5), which has been observed in various aggressive tumors, including melanoma, hepatocellular carcinoma (HCC), breast and ovarian cancer (5-8). Tumors that exhibit VM tend to metastasize easily, develop advanced tumor stages and high tumor grades, and present with a lower survival rate (9). Factors involved in regulating VM formation and stability, such as ephrin-A2 (EphA2), vascular endothelial cadherin (VE-cad) and matrix metalloproteinase (MMPs) also participate in tumor metastasis (10). According to their morphology, function and immunological factors, trophoblast cells could be divided into three types during embryogenesis and differentiation: cytotrophoblasts (CTBs), syncytiotrophoblasts (STBs) and intermediate trophoblasts (ITBs). CTBs are undifferentiated and proliferate to give rise to STBs and ITBs; thus, these 
cells are termed trophoblast stem cells, which identifies their function as tumor stem cells (11). Trophoblast stem cells participating in the formation of the placenta obtain endothelial cell phenotypes during the differentiation process (12) and are associated with a malignant tumor behavior (13). A previous morphological study of clinical CC tissues has demonstrated that pseudovascular channels are lined with STBs rather than CTBs or ITBs (14). These results suggested that trophoblast cells utilized VM to support tumor development in CC (14).

The cyclic adenosine monophosphate-protein kinase A (cAMP-PKA) signaling pathway serves an important role in regulating embryo implantation (15) combined with the Notch pathway and differentiation of endothelial cells (16). Forskolin is a typical activator of the cAMP pathway by direct activation of adenylate cyclase (17), and has been widely used as an inducer of syncytiolization and expression of invasion-associated molecules $(18,19)$. However, little is currently known about the role of this cAMP activator and its mechanism in VM formation in CC.

The epithelial-to-mesenchymal transition (EMT) is a process in which epithelial cells lose their epithelial features and simultaneously acquire mesenchymal features (20). EMT serves an important role in embryogenesis, wound healing, fibrosis and tumor metastasis (21), and has recently been demonstrated to be involved in VM formation in human HCC, breast and colon cancer $(7,22,23)$. The Notch signaling pathway is highly conserved and has been identified in arterial-venous specification (24). The Notch signaling pathway is activated by the interaction of trans-membrane ligands of the Jagged (Jagged1 and 2) and Delta (Delta-like 1, 3 and 4) families with Notch receptors (Notch1-4). Blockage of Notch in the endothelium permits unproductive angiogenesis and decelerates tumor growth (25). A previous study has demonstrated that Notch-1 signaling participates in the EMT process initiated by hypoxic conditions in CC cells (26).

The present study aimed to test the hypothesis that VM and metastatic ability would be affected during syncytiolization of cytotrophoblast induced by forskolin, and also to explore whether Notch-mediated EMT was involved in the regulation of these biological phenomena in vitro and in vivo.

\section{Materials and methods}

Cell culture and reagents. Human CC cell lines JEG-3 (derived from a gestational CC) and JAR (derived from a male fetus CC) were purchased from the American Type Culture Collection, and the human umbilical vein endothelial cell (HUVEC) line was kindly provided by Dr Jer-Tsong Hsieh (University of Texas Southwestern Medical Center, Dallas, TX, USA). These cells were cultured in DMEM (Gibco; Thermo Fisher Scientific, Inc.) with $10 \%$ fetal bovine serum (Gibco; Thermo Fisher Scientific, Inc.). All cells were cultured at $37^{\circ} \mathrm{C}$ in a humidified atmosphere with $5 \% \mathrm{CO}_{2}$. The cells used in the present study were of the third generation after resuscitation and passage. When cells reached $50 \%$ confluence, they were treated with $100 \mu \mathrm{M}$ forskolin (Abcam) or the vehicle (0.1\% DMSO) for $48 \mathrm{~h}$ at $37^{\circ} \mathrm{C}$. DMSO was purchased from Sigma-Aldrich; Merck KGaA. Notch-1 signaling was blocked with $10 \mu \mathrm{M}$ DAPT (Abcam) for $24 \mathrm{~h}$.

Wound healing assay. JAR and JEG-3 cells treated with forskolin or DMSO were scratched in 6-well plates with a
$200 \mu 1$ pipette tip when the cell confluence reached $\sim 90 \%$. Cells were incubated in serum-free medium (SFM), and the wound was photographed at 0 and $24 \mathrm{~h}$ under a phase-contrast microscope (x10 magnification; Olympus Corporation) and the total wound area was measured.

Transwell migration and invasion assays. The effects of forskolin on cell migratory and invasive abilities were assessed using 8- $\mu \mathrm{m}$-pore Transwell inserts (EMD Millipore). For the migration assay, a $200 \mu \mathrm{l}$ SFM suspension with $5 \times 10^{4}$ JAR or JEG-3 cells pre-incubated with forskolin or DMSO for $48 \mathrm{~h}$ were added to the upper chambers. The lower chambers were filled with $800 \mu \mathrm{l}$ medium containing $10 \%$ fetal bovine serum. For cell invasion assay, the upper chambers were pre-coated with $50 \mu$ l Matrigel (Sigma-Aldrich; Merck KGaA) for 4 h. A $200 \mu \mathrm{l}$ SFM suspension with $8 \times 10^{4}$ JAR or $5 \times 10^{4}$ JEG-3 cells pre-incubated with forskolin or DMSO for $48 \mathrm{~h}$ were added to the upper chambers. For the HUVEC migration assay, $200 \mu \mathrm{l}$ HUVECs suspended in SFM at $2 \times 10^{5}$ cells $/ \mathrm{ml}$ were seeded into the upper chamber, and $1 \times 10^{4} \mathrm{CC}$ cells pretreated with forskolin or DMSO for $48 \mathrm{~h}$ were added to the lower chamber. Following 24-h incubation at $37^{\circ} \mathrm{C}$, cells that had migrated to the lower chamber were fixed with $4 \%$ paraformaldehyde for $25 \mathrm{~min}$ and stained with crystal violet for $10 \mathrm{~min}$ at room temperature. The upper chamber was wiped with a cotton swab, and cell numbers were counted in five randomly selected fields per well under a phase-contrast microscope (x200 magnification; Olympus Corporation).

$3 D$ cultures and tubule formation. The assay was performed as previously described by Zhan et al $(6,27)$. A total of $200 \mu \mathrm{l}$ Matrigel (BD Biosciences) was added to 24-well plates and incubated at $37^{\circ} \mathrm{C}$ for $1 \mathrm{~h}$. CC cells $\left(1 \times 10^{5}\right.$ cells/well) suspended in SFM were seeded into Matrigel-coated wells following treatment with forskolin or DMSO for $48 \mathrm{~h}$. After 6 and $24 \mathrm{~h}$, tubule structure formation was observed, and the number and completeness of the tubule were assessed under a phase-contrast microscope (x200 magnification; Olympus Corporation).

Reverse transcription-quantitative PCR (RT-qPCR). Total RNA was harvested using the TRIzol ${ }^{\circledR}$ reagent (Thermo Fisher Scientific, Inc.) according to the manufacturer's protocol and quantified by spectrophotometry. The samples were reverse-transcribed to cDNA using the PrimeScript ${ }^{\mathrm{TM}}$ RT Reagent kit (Takara Bio, Inc.). The RNA sample was incubated with $2 \mu \mathrm{l} 5 \mathrm{X}$ PrimeScript RT Master Mix at $37^{\circ} \mathrm{C}$ for $15 \mathrm{~min}$, followed by $85^{\circ} \mathrm{C}$ for $5 \mathrm{sec}$ and terminated at $4^{\circ} \mathrm{C}$. QPCR was performed using the SYBR ${ }^{\circledR}$ Green PCR Master Mix (Takara Bio, Inc.) on a CFX96 real-time PCR system (Bio-Rad Laboratories, Inc.). The PCR protocol was $94^{\circ} \mathrm{C}$ for $10 \mathrm{~min}$, followed by 40 cycles of $94^{\circ} \mathrm{C}$ for $10 \mathrm{sec}$ and $60^{\circ} \mathrm{C}$ for $30 \mathrm{sec}$. The primer sequences used in the present study are listed in Table I. Data were normalized to GAPDH expression and all reactions were performed in triplicate. Relative gene expression was calculated using the $2^{-\Delta \Delta \mathrm{Cq}}$ method (28).

Western blot analysis. Cells at 80-90\% confluence were washed three times with cold PBS and lysed in RIPA buffer $(50 \mathrm{mM}$ Tris pH 8.0, $150 \mathrm{mM} \mathrm{NaCl}, 0.1 \%$ SDS, $1 \%$ NP-40, and $0.5 \%$ 
Table I. Quantitative PCR primers.

\begin{tabular}{|c|c|}
\hline Gene & Sequences $\left(5^{\prime} \rightarrow 3^{\prime}\right)$ \\
\hline \multirow[t]{2}{*}{ GAPDH } & F: ATGGGGAAGGTGAAGGTCGG \\
\hline & R: CACGGTGCCATGGAATTTGC \\
\hline \multirow[t]{2}{*}{ VE-cad } & F: GCGACTACCAGGACGCTTTCA \\
\hline & R: CATGTATCGGAGGTCGATGGTG \\
\hline \multirow[t]{2}{*}{ EphA2 } & F:CTCTCACACCCCGTATGGCAAAC \\
\hline & R: TCCTGGTCGCCAGACATCAC \\
\hline \multirow[t]{2}{*}{ MMP2 } & F: TACAGGATCATTGGCTACACACC \\
\hline & R: GGTCACATCGCTCCAGACT \\
\hline \multirow[t]{2}{*}{ MMP9 } & F: TGTACCGCTATGGTTACACTCG \\
\hline & R: GGCAGGGACAGTTGCTTCT \\
\hline \multirow[t]{2}{*}{ E-cadherin } & F: GGGCTCAAGTGACTCGTAACGA \\
\hline & R: CAGCCGCTTTCAGATTTTCATC \\
\hline \multirow[t]{2}{*}{ CK19 } & F: AACGGCGAGCTAGAGGTGA \\
\hline & R: GGATGGTCGTGTAGTAGTGGC \\
\hline \multirow[t]{2}{*}{ N-cadherin } & F: ATGAAAGACCCATCCACG \\
\hline & R: CCTGCTCACCACCACTA \\
\hline \multirow[t]{2}{*}{ Vimentin } & F: CTTCCGCGCCTACGCCA \\
\hline & R: GCCCAGGCGAGGTACTCC \\
\hline \multirow[t]{2}{*}{ ZEB1 } & F: GAAAGTGATCCAGCCAAATGGA \\
\hline & R: TTTGGGCGGTGTAGAATCAGAG \\
\hline \multirow[t]{2}{*}{ HES 1} & F: CTTCCGCGCCTACGCCA \\
\hline & R: GCCCAGGCGAGGTACTCC \\
\hline \multirow[t]{2}{*}{ NICD } & F: GGAGGCATCCTACCCTTTTC \\
\hline & R: TGTGTTGCTGGAGCTTCTTC \\
\hline
\end{tabular}

VE-cad, vascular endothelial cadherin; EphA2, ephrin-A2; MMP, matrix metalloproteinase; CK19, cytokeratin-19; ZEB1, zinc finger E-box-binding homeobox 1; HES1, Hes family BHLH transcription factor 1 ; NICD, Notch 1 .

sodium deoxycholate) containing protease inhibitors $(1 \mathrm{mM}$ PMSF; Sigma-Aldrich; Merck KGaA). Protein samples were quantified by bicinchoninic acid assay, and $20 \mu \mathrm{g}$ of protein was loaded per lane, separated by SDS-PAGE (8-12\% gel) and electrophoretically transferred to nitrocellulose membranes (EMD Millipore). The membranes were blocked with 5\% skimmed milk at room temperature for $1 \mathrm{~h}$ and incubated with primary antibodies against GAPDH (cat. no. KG-5G4; Kangchen; 1:1,000), syncytin-1 (cat. no. SC130888; Santa Cruz; 1:200), VEGFA (cat. no. ab185265; Abcam; 1:1,000), VE-cadherin (VE-cad; cat. no. ab205336; Abcam; 1:1,000), MMP-2 (cat. no. ab37150; Abcam; 1:1,000), Hes family BHLH transcription factor 1 (HES1; cat. no. ab108937; Abcam; 1:500), cleaved Notch1 (NICD; cat. no. 4147; Cell Signaling Technology, Inc.; 1:1,000), N-cadherin (N-cad; cat. no. 13116; Cell Signaling Technology, Inc.; 1:500), zinc finger E-box-binding homeobox 1 (ZEB1; cat. no. 3396; Cell Signaling Technology, Inc.; 1:1,000), E-cadherin (E-cad; cat. no. sc71009; Santa Cruz Biotechnology, Inc.; 1:1,000), cytokeratin (CK19; cat. no. sc376126; Santa Cruz Biotechnology, Inc.; 1:1,000), vimentin (cat. no. sc80975; Santa Cruz Biotechnology, Inc.; 1:400) and MMP-9 (cat. no. sc10737; Santa Cruz Biotechnology, Inc.; 1:1,000) at $4^{\circ} \mathrm{C}$ overnight, followed

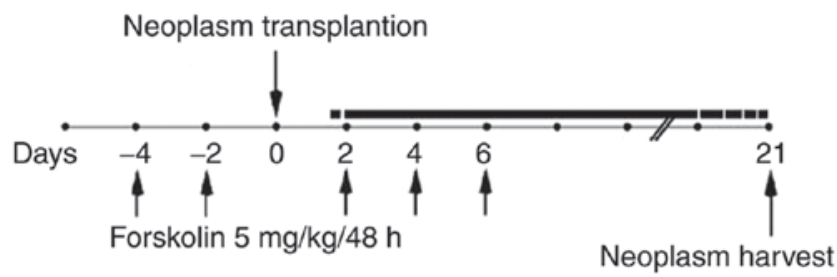

Figure 1. Xenograft tumor model. The forskolin groups were injected intraperitoneally with $5 \mathrm{mg} / \mathrm{kg}$ forskolin on days -4, -2, 2, 4 and 6; JEG-3 cells were injected subcutaneously into nude mice on day 0 , and xenograft tumors were harvested after 3 weeks.

by washing with TBS containing $0.1 \%$ Tween-20 and $1 \mathrm{~h}$ incubation with a horseradish peroxidase (HRP)-conjugated goat anti-rabbit $\operatorname{IgG}(\mathrm{H}+\mathrm{L})$ secondary antibody (cat. no. sc2301; Santa Cruz Biotechnology, Inc.; 1:800) at room temperature. Protein bands were visualized using the Odyssey detection system (LI-COR Biosciences) and quantified using ImageLab software (version 4.1; Bio-Rad Laboratories, Inc.).

Xenograft tumor model. Animal care and protocols were performed according to the guidelines of the Institutional Animal Care and Use Committee of Xi'an Jiaotong University. A preliminary experiment indicated that xenografts in nude mice were easier to establish using JEG-3 cells compared with JAR cells (29); therefore, JEG-3 cells were used for the in vivo study. A total of 12 -week-old athymic nude mice (Charles River Laboratories, Inc.) were randomly divided into two groups; the mice in the forskolin group were injected intraperitoneally with $5 \mathrm{mg} / \mathrm{kg}$ forskolin diluted in a PBS/DMSO solution (15:0.1) five times on days -4, -2, 2, 4 and 6 (relative to JEG-3 cell injection), and the control groups were injected with equal volumes of DMSO (30). A total of 5x10 ${ }^{6} \mathrm{JEG}-3$ cells were injected subcutaneously into nude mice to establish the xenograft model on day 0 . Xenograft tumors were harvested after 3 weeks and used for western blotting, hematoxylin and eosin (H\&E) staining and CD31/periodic acid-Schiff (PAS) double staining (Fig. 1).

CD31/PAS double staining. Immunohistochemistry was performed on 4- $\mu$ m-thick sections. Sections were deparaffinized using xylene and dehydrated using ethanol (100, 95, 80, 70 and $50 \%$ for $3 \mathrm{~min}$ each). Antigen retrieval was performed using citric acid for $5 \mathrm{~min}$ at $121^{\circ} \mathrm{C}$. After blocking with $3 \%$ hydrogen peroxide for $30 \mathrm{~min}$ at room temperature, the sections were incubated with an anti-CD31 antibody (cat. no. ab28364; Abcam; $1: 100$ ) overnight at $4^{\circ} \mathrm{C}$ and anti-rabbit ChemMate ${ }^{\mathrm{TM}}$ EnVision ${ }^{\mathrm{TM}} / \mathrm{HRP}$ secondary antibody for $1 \mathrm{~h}$ at room temperature, followed by staining with DAB for $40 \mathrm{sec}$ (cat. no. GK500705; Dako; Agilent Technologies, Inc.). The sections were incubated with PAS $(0.5 \%$ periodic acid for $10 \mathrm{~min}$ and Schiff solution for $15 \mathrm{~min}$ ) at room temperature prior to $\mathrm{H} \& \mathrm{E}$ counterstaining (hematoxylin for $5 \mathrm{~min}$ and $0.5 \%$ eosin for $1-3 \mathrm{~min})$. VM structures were defined as CD31-negative PAS-positive structures.

Statistical analysis. Data are presented as the mean \pm standard error of the mean of at least three independent experiments. GraphPad Prism software (version 6.0; GraphPad Software, 

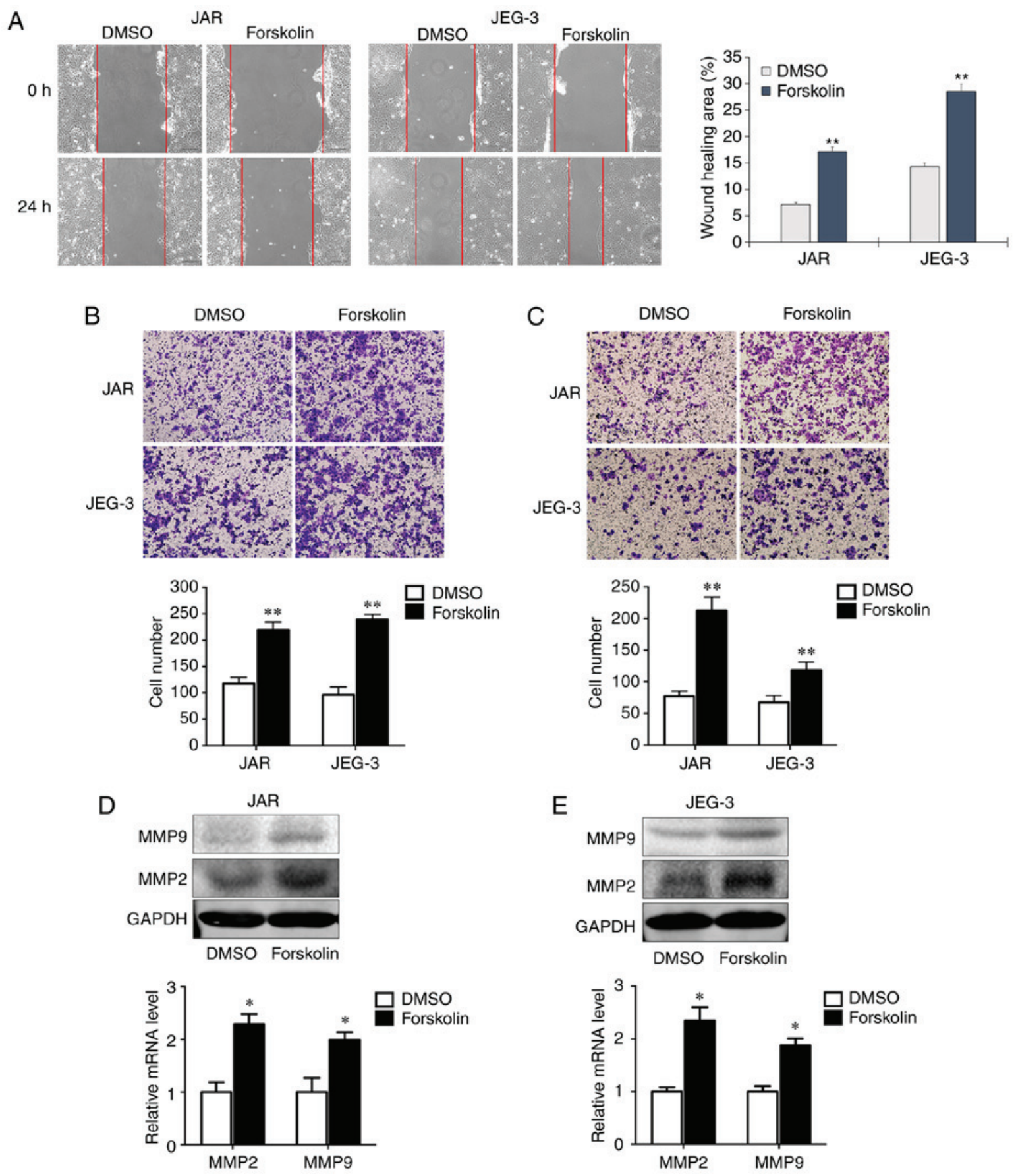

Figure 2. Forskolin promotes CC cell invasion and migration in vitro. (A-C) The effects of forskolin on CC cell migration and invasion were examined by (A) wound healing, (B) Transwell migration and (C) Transwell invasion assays. (D and E) Forskolin increased the expression of MMP2 and MMP9 in (D) JAR and (E) JEG-3 cells, as detected by western blotting and reverse transcription-quantitative PCR. ${ }^{*} \mathrm{P}<0.05,{ }^{* *} \mathrm{P}<0.01$ vs. DMSO. CC, choriocarcinoma; MMP, matrix metalloproteinase.

Inc.) was used to analyze differences between two groups with Student's t-test, and among multiple groups with one-way ANOVA followed by Tukey's post hoc test. $\mathrm{P}<0.05$ was considered to indicate a statistically significant difference.

\section{Results}

Forskolin promotes CC cell invasion and migration in vitro. Cell motility is associated with tumor metastasis. In order to assess whether forskolin could stimulate the invasion and migration of JAR and JEG-3 cells, the present study used wound healing and transwell assays. In the wound healing assay, the migration rates of the forskolin groups at $24 \mathrm{~h}$ were significantly higher compared with those of the DMSO groups $(\mathrm{P}<0.01 ;$ Fig. $2 \mathrm{~A})$. In the migration assay presented in Fig. $2 \mathrm{~B}$, the numbers of migrant cells were $\sim 2$-fold higher compared with the control groups when JAR and JEG-3 cells were treated with forskolin $(\mathrm{P}<0.01)$. Similarly, the invasive capacity was markedly enhanced when cells treated with forskolin compared with the respective control groups (P<0.01; Fig. 2C). Consistently, RT-qPCR and western blot assays demonstrated significantly increased MMP2 and 
A
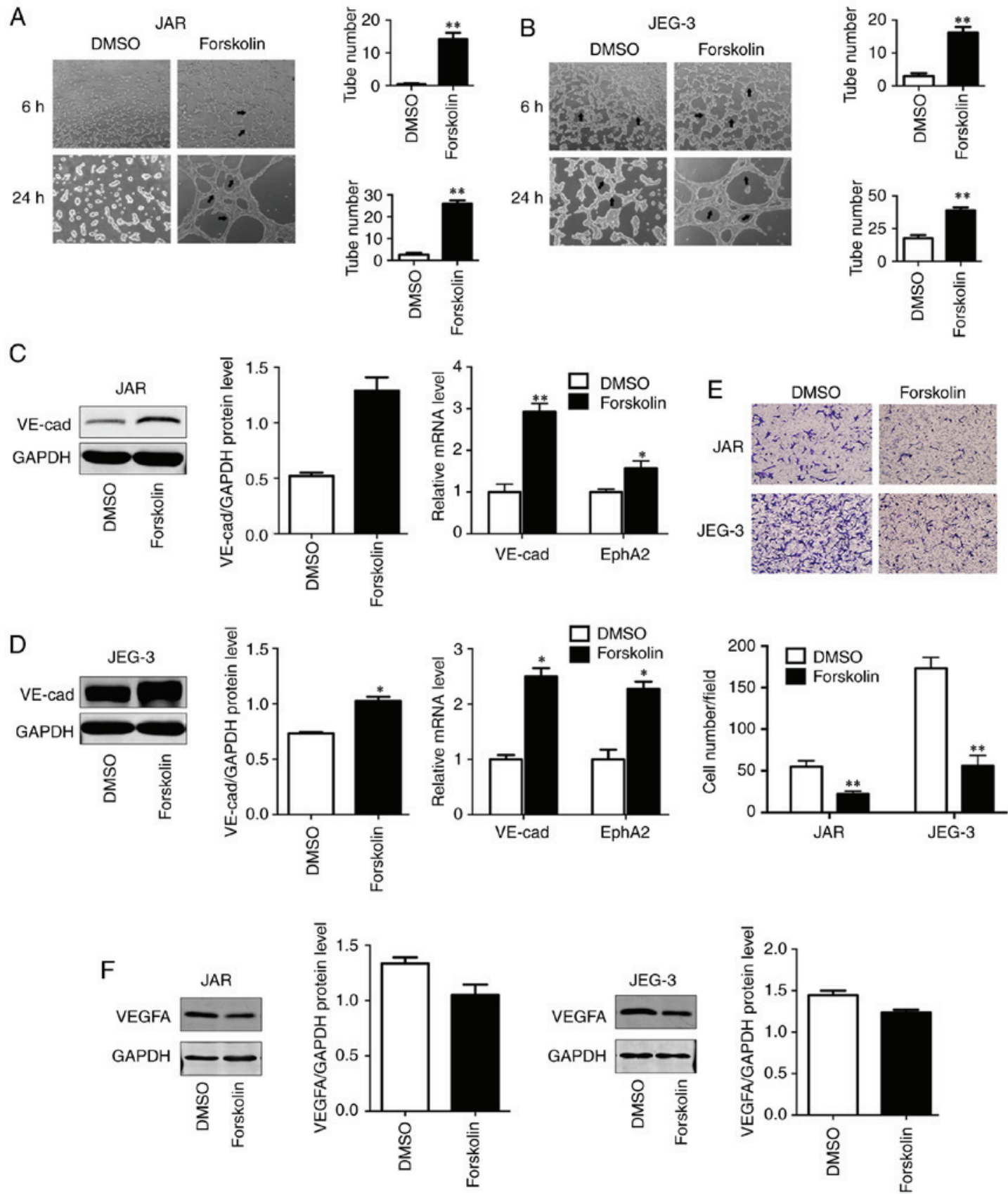

Figure 3. Forskolin promotes VM formation of choriocarcinoma cells and decreases the recruitment of HUVECs in vitro. (A and B) Forskolin enhanced the tube formation capacity of (A) JAR and (B) JEG-3 cells, and this capacity was lower in JAR cells. (C and D) Western blotting was used to detect the expression of VE-cad at the protein level; reverse transcription-quantitative PCR was used to detected the expression of VE-cad and EphA2 at the mRNA level in (C) JAR and (D) JEG-3 cells. (E) Forskolin decreased the recruitment of HUVECs in JAR and JEG-3 cells. (F) VEGFA protein level was detected by western blot analysis. ${ }^{*} \mathrm{P}<0.05,{ }^{* *} \mathrm{P}<0.01$ vs. DMSO. VM, vasculogenic mimicry; HUVECs, human umbilical vein endothelial cells; VE-cad, vascular endothelial cadherin; EphA2, ephrin-A2; VEGFA, vascular endothelial growth factor $\alpha$.

MMP9 expression following forskolin treatment in the two $\mathrm{CC}$ cell lines compared with the corresponding controls (Fig. 2D and E).

Forskolin promotes VM formation of CC cells in vitro. VM has been demonstrated to be associated with cell migration, invasion and capillary tube formation (6). In order to investigate the role of forskolin in VM, the present study used a well-established in vitro model of $3 \mathrm{D}$ culture. Compared with the control group, JAR and JEG-3 cells treated with forskolin exhibited an enhanced capability of forming typical capillary-like structures on 3D Matrigel medium $(\mathrm{P}<0.01$; Fig. 3A and B), which appeared more typical in JEG-3. As VE-cad and EphA2 act in a coordinated manner as key regulatory elements during the process of VM (31), these two VM-associated markers were detected via western blotting or RT-qPCR; both VE-cad protein levels and EphA2 mRNA levels were upregulated in the forskolin-treated group compared with the control group $(\mathrm{P}<0.01$; Fig. $3 \mathrm{C}$; $\mathrm{P}<0.05$; Fig. 3D), suggesting that forskolin was involved in the formation of vasculogenic-like networks in CC cells. In addition, to reveal the potential roles of forskolin in angiogenesis, an 

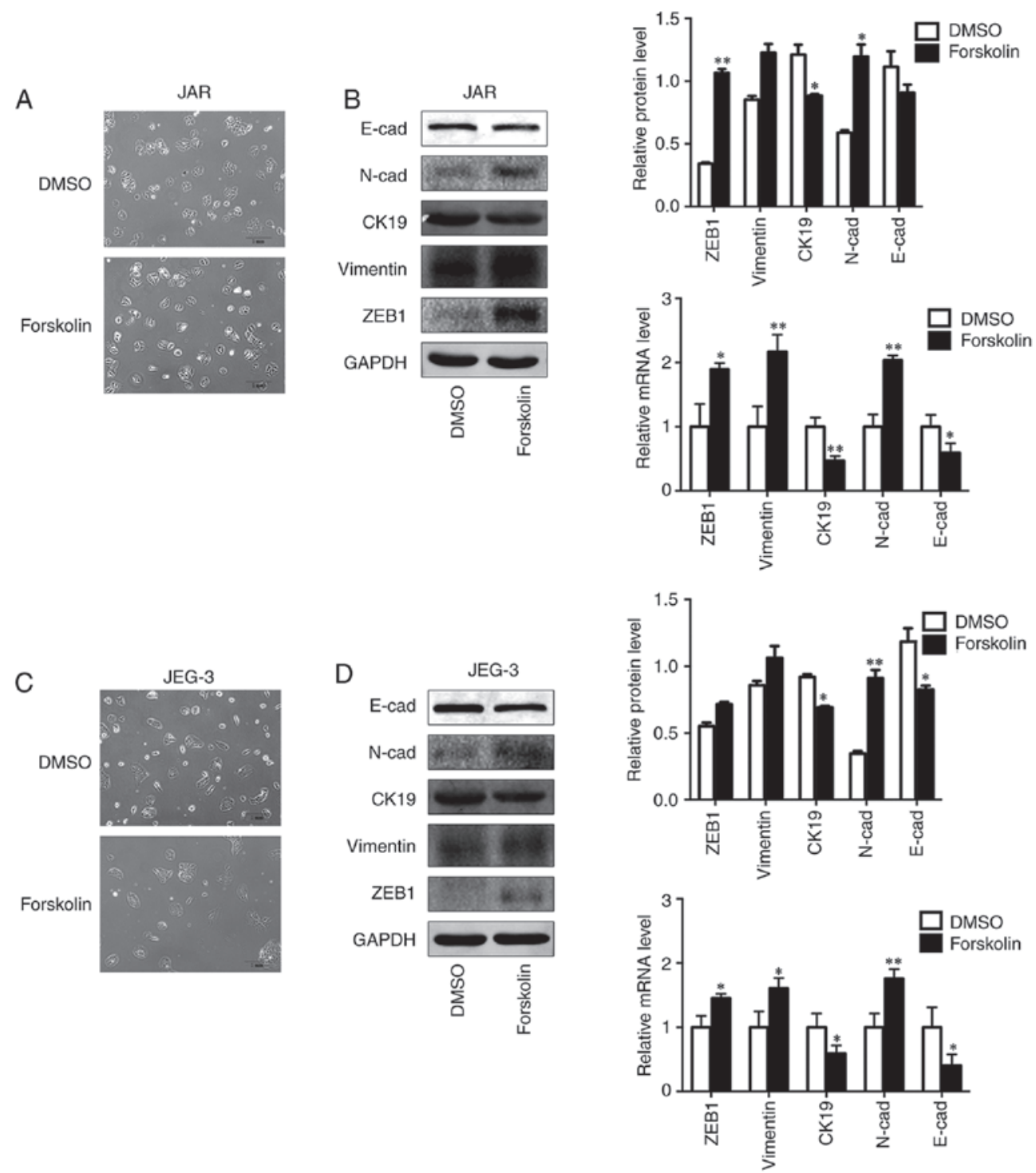

Figure 4. Forskolin induces EMT of CC cells in vitro. (A and C) Forskolin induced the morphological changes of JAR and JEG-3 cells from cobblestone-like to spindle-like. (B) Western blot and RT-qPCR was used to show the expression of EMT markers in forskolin-treated JAR cells. (D) Western blot and RT-qPCR was used to show the expression of EMT markers in forskolin-treated JEG-3 cells. ${ }^{*} \mathrm{P}<0.05,{ }^{* *} \mathrm{P}<0.01$. CC, choriocarcinoma; EMT, epithelial-to-mesenchymal transition; RT-qPCR, reverse transcription-quantitative PCR.
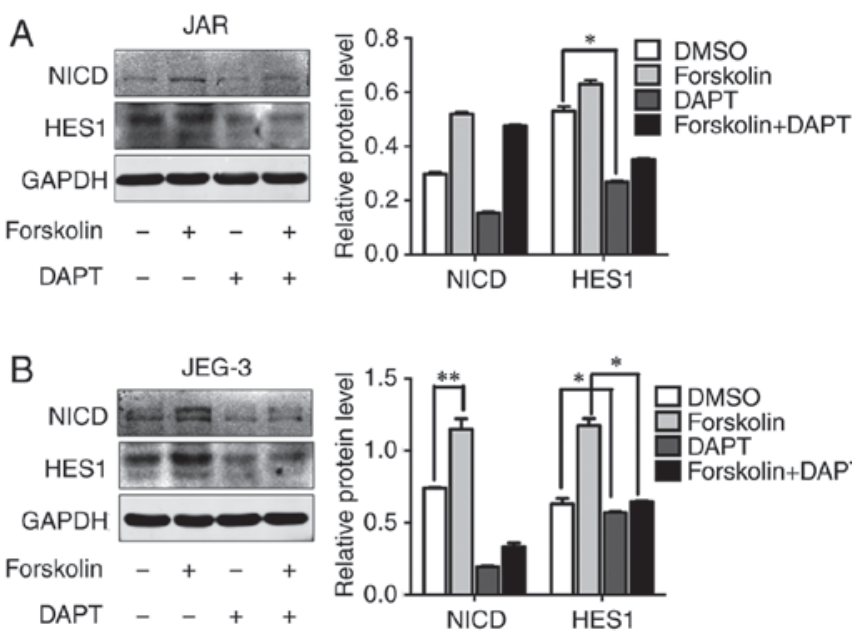
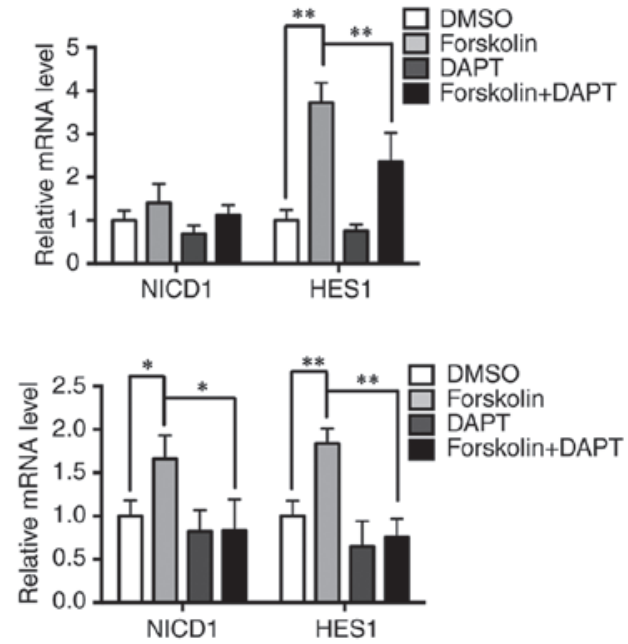

Figure 5. Notch signaling is activated in forskolin-treated CC cells, which can be reversed by DAPT. (A and B) Western blotting and reverse transcription-quantitative PCR revealed the expression of NICD and HES1 in (A) JAR and (B) JEG-3 cells. "P<0.05, " P $<0.01$. CC, choriocarcinoma; NICD, Notch 1; HES1, Hes family BHLH transcription factor 1. 

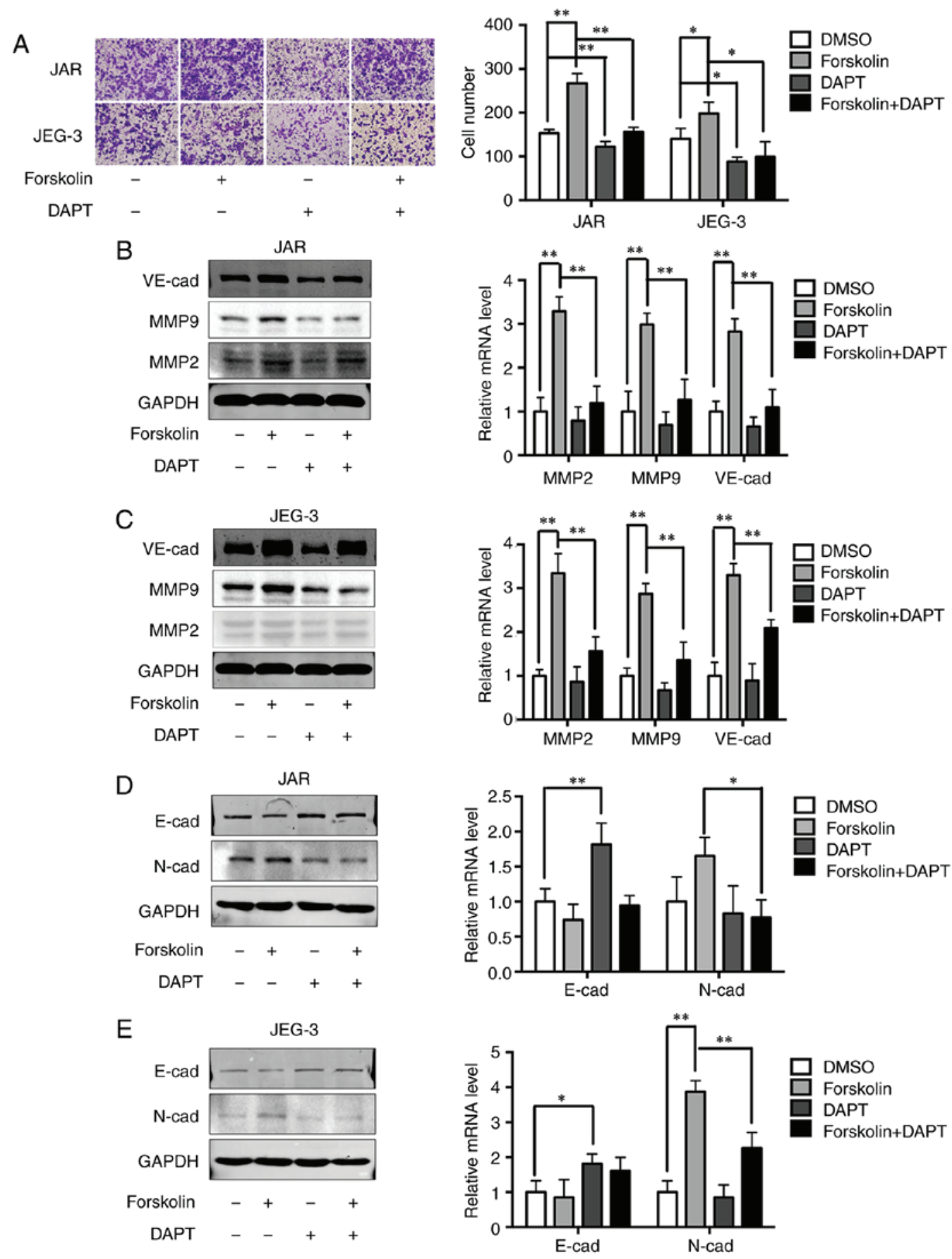

Figure 6. DAPT reverses CC cell invasion, VM and EMT by suppressing Notch signaling. (A) Transwell migration results of the changes in migratory ability of JAR and JEG-3 cells following blocking the Notch signaling pathway. (B and C) DAPT reversed the enhancement of MMP2, MMP9 and VE-cad in forskolin and DMSO-treated (B) JAR and (C) JEG-3 cells. (D and E) DAPT reversed EMT in forskolin and DMSO-treated (D) JAR and (E) JEG-3cells. "P<0.05, ${ }^{* *} \mathrm{P}<0.01$. VM, vasculogenic mimicry; EMT, epithelial-to-mesenchymal transition; MMP, matrix metalloproteinase; VE-cad, vascular endothelial cadherin.

endothelial recruitment assay was performed, which revealed a decreased ability of forskolin-treated CC cells to recruit HUVECs compared with that of DMSO-treated cells $(\mathrm{P}<0.01$; Fig. 3E). In line with these results, the expression of vascular endothelial growth factor $\alpha$ (VEGFA), which serves a key role in tumor angiogenesis (32), was also effectively inhibited by forskolin treatment (Fig. 3F).

Forskolin induces EMT of CC cells in vitro. In order to investigate the biological variation of trophoblasts during syncytiolization induced by forskolin in CC cells, the morphological changes and EMT markers were observed and detected in the present study. The results demonstrated that the cells were converted to a spindle-like shape and lost cellular cohesiveness following administration of forskolin for $48 \mathrm{~h}$ (Fig. 4A and C), and the expression levels of epithelial markers E-cad and CK19 were significantly downregulated, whereas those of mesenchymal markers, including N-cad, Vimentin and ZEB1, were significantly upregulated (Fig. 4B and D). 


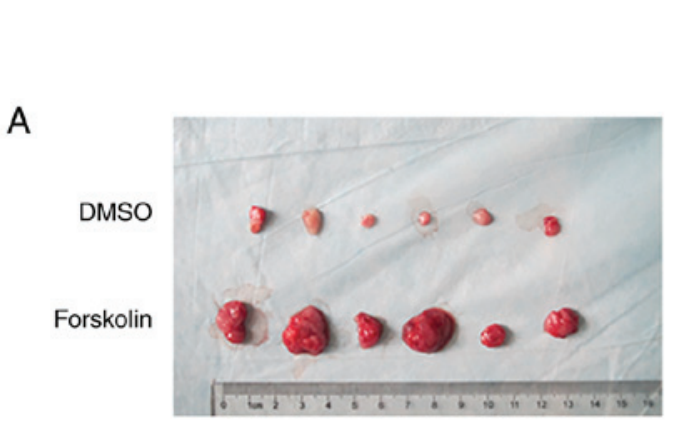

B
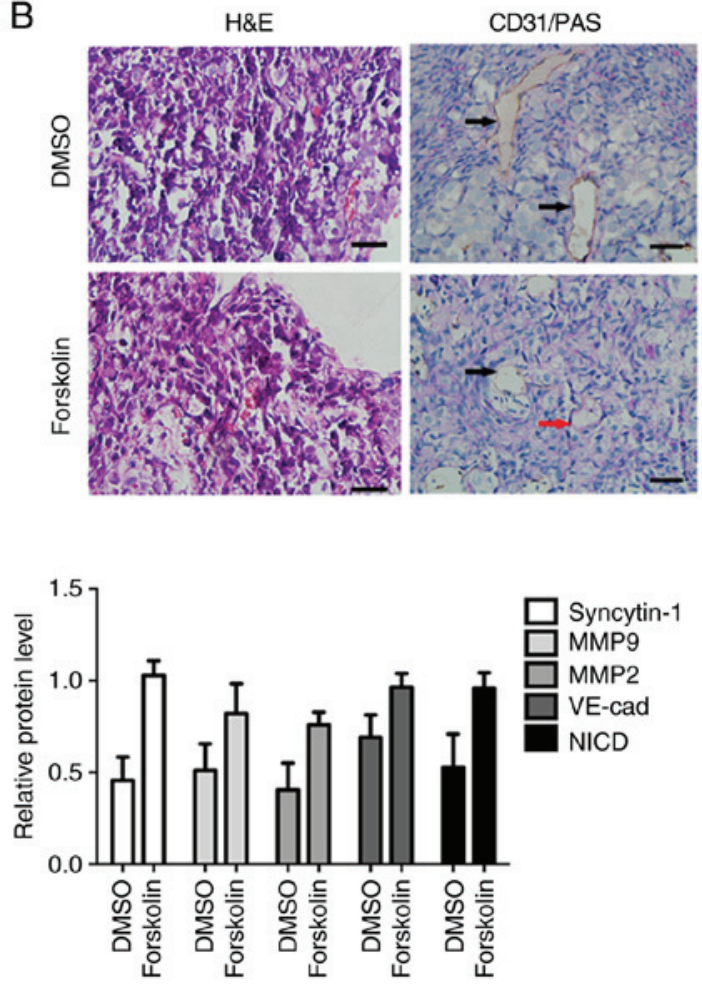

Figure 7. Forskolin promotes VM formation and metastasis in vivo. (A) Tumor size of forskolin or DMSO treated xenografts. (B) H\&E staining revealed capillary-like structure in subcutaneous xenografts; CD31/PAS double staining demonstrated VM formation in subcutaneous xenografts. Red arrows indicate capillary tubules formed by tumor cells; black arrows indicate endothelium-dependent vessels. (C) Western blotting was used to analyze MMP2, MMP9, VE-cad and NICD expression levels in forskolin- or DMSO-treated xenografts. VM, vasculogenic mimicry; PAS, periodic acid-Schiff; MMP, matrix metalloproteinase; VE-cad, vascular endothelial cadherin; NICD, Notch 1.

Notch-mediated EMT is activated in forskolin-induced syncytiolization in vitro. A previous study has demonstrated that Notch-1 signaling is involved in gestational trophoblastic neoplasia (26). Based on the result that the expression levels of NICD and HES1 were upregulated after forskolin treatment, $10 \mu \mathrm{M}$ DAPT was added into the culture medium to evaluate whether the Notch-1 signaling pathway was involved in regulating metastatic and VM capabilities in CC cells (Fig. 5A and B). In addition, DAPT could reverse the upregulating of NICD and HES. Consistently, inhibition of the Notch-1 signaling pathway also significantly attenuated the invasive ability of cells in the forskolin and DMSO groups compared with cells without DAPT treatment (Fig. 6A). Upregulation of MMP2, MMP9 and VE-cad expression levels by forskolin was also reversed by DAPT $(\mathrm{P}<0.01$; Fig. 6B and C). Changes in EMT-associated proteins were also evaluated following DAPT interference. Inhibition of the Notch-1 signaling pathway partially reversed the downregulation of E-cad and significantly reversed the upregulation of $\mathrm{N}$-cad in forskolin-treated cells (Fig. 6D and E), which indicated that the Notch-1 signaling pathway may mediate EMT during the syncytiolization process induced by forskolin.

Forskolin promotes cell proliferation and VM formation in vivo. In order to validate the function of forskolin in vivo, JEG-3 cells were subcutaneously implanted into the flanks of nude mice. After 3 weeks, the subcutaneous tumor diameter in the forskolin group was larger compared with that in the DMSO group (Fig. 7A). Tumor sections were stained with CD31 to observe tumor angiogenesis by counting the number of vessels; VM structures formed by tumor cells exhibited a PAS-positive basement membrane (25). Endothelium-dependent vessels and VM channels were observed in the excised tumors; PAS-positive tubular structures lined with CD31-negative cells were indicated, and intratumoral blood vessels were detected in all specimens (Fig. 7B). Consistent with the observation in vitro, forskolin treatment resulted in high expression of MMP2, MMP-9 and VE-cad, as well as the activation of the Notch-1 signaling pathway (Fig. 7C).

\section{Discussion}

$\mathrm{CC}$ is an aggressive solid tumor, which is characterized by abundant blood supply and earlier blood metastasis compared with other female genital tumors, and can develop a vascular system containing fragile blood vessels (16). VM is a pattern of tumor micro-circulation important for the growth and progression of solid tumors (5-8). VM was initially reported in melanomas and occasionally in other solid tumors, including breast, ovarian and prostate cancer $(5-8,32)$. Channels in VM are similar to the embryonic microcirculatory networks (33). The capacity of STBs to perform endothelium-like functions, such as controlling substrate exchange across the villous surface, has been well established $(14,34)$. A previous study of VM revealed that tumor cells established their own pseudovascular networks by STBs in clinical tissues from patients with CC (14). Our previous study demonstrated that compared with normal villus, the level of syncytiolization in 
$\mathrm{CC}$ tissues was higher and associated with the malignancy of gestational trophoblastic disease (Supplementary Material). However, little is currently known about the role of syncytiolization of cytotrophoblast cells in VM formation. Forskolin, an activator of the cAMP pathway, is widely used as an inducer of syncytiolization of trophoblasts; it elevates cAMP levels and causes accumulation of VE-cad inside the cell-cell junctions on HUVECs (35). VE-cad and EphA2 act in a coordinated manner as a key element in the process of VM (31). To assess whether VM occurred in the process of syncytiolization and the underlying mechanisms, CC cell lines JEG-3 and JAR were treated with $100 \mu \mathrm{M}$ forskolin for $48 \mathrm{~h}$ in the present study, and a positive association between forskolin and VM was observed. Compared with the control group cells treated with DMSO, forskolin not only enhanced the migratory and invasive abilities of CC cells, but also promoted VM formation and decreased tube formation detected by a 3D system accompanied by increased VE-cad and EphA2 expression. The behavior of trophoblasts is similar to aggressive tumors during differentiation and implantation (36), and excessive fusion of cytotrophoblasts to syncytiotrophoblasts leads to gestational trophoblastic neoplasia, including CC $(37,38)$. Trophoblasts are highly invasive due to the secretion of extracellular proteases, such as MMPs. MMP2 and MMP9 are key enzymes associated with trophoblast invasion $(39,40)$ and malignancy $(41,42)$. In the present study, increases in MMP-2 and MMP-9 expression were observed in forskolin-treated cells compared with the control cells, which was consistent with high cell invasiveness.

Previously research has revealed that VM could be observed in surgical specimens from patients with CC (14). In the present study, animal xenograft tumor models also exhibited enhanced function of proliferation and VM in vivo, and intratumoral blood vessels were easily detected. This may have resulted in part from the instability of forskolin concentration in local tumors in vivo, and these results may explain why blood metastasis is more easily observed in patients with CC. Forskolin can promote the differentiation of CTB to STB and the formation of VM structure, and it is reported that red blood cells are surrounded by STB in CC cells without endothelial cells (14). In addition, the blood supply by VM may not be as effective as intratumoral blood vessels, which may explain why $\mathrm{CC}$ tissue is characterized by massive tumor necrosis and hemorrhage.

EMT is a crucial process in cancer progression and is closely associated with the remodeling of vascular endothelial cells (43). Epithelial tumor cells with the capacity of VM exhibit certain endothelial phenotypes of mesenchymal cells, which are similar to the EMT process (44). To comprehensively understand the formation of VM mechanism during forskolin-induced differentiation of trophoblasts, the present study evaluated the switch of EMT. The results revealed that forskolin significantly decreased the expression of epithelial markers and enhanced the expression of mesenchymal markers, which was in agreement with the results of a previous study (44). These findings suggested that forskolin enhanced the formation of VM channels via the induction of EMT.

Notch signaling influences trophoblastic differentiation $(45,46)$ and vascular development in several types of human cancer, including CC (25). Notch-1 knockouts are lethal for the embryo due to vascular and somatic defects (47), and activation of Notch can enhance cell differentiation by upregulating anti-apoptotic genes (48). The present study aimed to investigate the signaling pathway involved in EMT during forskolin-induced syncytiolization. Increased expression levels of Notch-1 signaling markers were observed in CC cells treated with forskolin, suggesting that this process was under the control of the Notch signaling pathway. Since Notch-1 activation is dependent on $\gamma$-secretase, the present study utilized DAPT to block the activation of Notch-1 and assessed the NICD- and Notch-1-targeting genes. HES1 was significantly decreased, along with the attenuation in invasion and VM markers. The invasive ability and VM formation was weakened and the process of EMT was reversed by DAPT. In addition, our previous study also demonstrated that overexpression of hypoxia-inducible factor $1 \alpha$ induced migration and invasion through Notch signaling (26). Therefore, the inhibitor of Notch signaling pathway maybe used to block the syncytiolization of trophoblast cells indirectly, although the side effects and exact functions need to be determined in further research.

In conclusion, the present study revealed that forskolin may act as a tumor promoter in CC through enhancing cell metastasis and VM formation via the activation of Notch-mediated EMT during syncytiolization. As forskolin induced CC syncytiolization and increased human chorionic gonadotrophin secretion, a cell model stably expressing hCG may be established to further study the crosstalk between Notch-1 signaling and hCG. The results of the present study provided an additional rationale to explain why massive hemorrhage and hematogenous metastasis commonly occur in patients with CC, which suggested that inhibition of syncytiolization ability of trophoblast cells may be an effective way to inhibit tumor metastasis.

\section{Acknowledgements}

The authors would like to thank Mr. XY Wang, Ms. S Xu and Mr. K Wang (Oncology Research Lab, Key Laboratory of Environment and Genes Related to Disease of the First Affiliated Hospital of Xi'an Jiaotong University, Xi'an, China) for providing technical support.

\section{Funding}

This work was supported by the National Natural Science Foundation of China (grant no. 81671491), the Shaanxi Natural Science Basic Research Program-General Projects (grant no. 2017JM8055) and the Science Foundation of The First Affiliated Hospital of Xi'an Jiaotong University (grant no. 2015YK8).

\section{Availability of data and materials}

All data generated or analyzed during this study are included in this published article.

\section{Authors' contributions}

YX, RS and RA conceived and designed the study, developed the methodology, wrote and reviewed the manuscript. YX, RS, LY and WZ acquired, analyzed and interpreted the data. 
YX and RA provided administrative, technical and material support and supervised the study. All authors read and approved the final version of the manuscript.

\section{Ethics approval and consent to participate}

All animal experiments and the use of human tissue samples were approved by Xi'an Jiaotong University Ethics Committee. Animal experiments were in accordance with the principles of Laboratory Animal Care and the Practice Guidelines for Laboratory Animals of China.

\section{Patient consent for publication}

Not applicable.

\section{Competing interests}

The authors declare that they have no competing interests.

\section{References}

1. Stevens FT, Katzorke N, Tempfer C, Kreimer U, Bizjak GI, Fleisch MC and Fehm TN: Gestational trophoblastic disorders: An update in 2015. Geburtshilfe Frauenheilkd 75: 1043-1050, 2015.

2. Abu-Rustum NR, Yashar CM, Bean S, Bradley K, Campos SM, Chon HS, Chu C, Cohn D, Crispens MA, Damast S, et al: Gestational trophoblastic neoplasia, version 2.2019, NCCN clinical practice guidelines in oncology. J Natl Compr Canc Netw 17: 1374-1391, 2019.

3. Alazzam M, Tidy J, Osborne R, Coleman R, Hancock BW and Lawrie TA: Chemotherapy for resistant or recurrent gestational trophoblastic neoplasia. Cochrane Database Syst Rev 13: CD008891, 2012.

4. Viallard C and Larrivee B: Tumor angiogenesis and vascular normalization: Alternative therapeutic targets. Angiogenesis 20: 409-426, 2017.

5. Maniotis AJ, Folberg R, Hess A, Seftor EA, Gardner LM, Pe'er J, Trent JM, Meltzer PS and Hendrix MJ: Vascular channel formation by human melanoma cells in vivo and in vitro: Vasculogenic mimicry. Am J Pathol 155: 739-752, 1999.

6. Zhao N, Sun H, Sun B, Zhu D, Zhao X, Wang Y, Gu Q, Dong X, Liu F, Zhang Y and Li X: miR-27a-3p suppresses tumor metastasis and VM by down-regulating VE-cadherin expression and inhibiting EMT: An essential role for Twist-1 in HCC. Sci Rep 6 : 23091, 2016.

7. Zhang D, Sun B, Zhao X, Ma Y, Ji R, Gu Q, Dong X, Li J, Liu F, Jia X, et al: Twist1 expression induced by sunitinib accelerates tumor cell vasculogenic mimicry by increasing the population of CD133+ cells in triple-negative breast cancer. Mol Cancer 13: 207, 2014.

8. Su M, Fan C, Gao S, Shen A, Wang X and Zhang Y: An HCG-rich microenvironment contributes to ovarian cancer cell differentiation into endothelioid cells in a three-dimensional culture system. Oncol Rep 34: 2395-2402, 2015.

9. Zhang J, Qiao L, Liang N, Xie J, Luo H, Deng G and Zhang J: Vasculogenic mimicry and tumor metastasis. J BUON 21 : 533-541, 2016

10. Qiao L, Liang N, Zhang J, Xie J, Liu F, Xu D, Yu X and Tian Y: Advanced research on vasculogenic mimicry in cancer. J Cell Mol Med 19: 315-326, 2015.

11. Okae H, Toh H, Sato T, Hiura H, Takahashi S, Shirane K, Kabayama Y, Suyama M, Sasaki H and Arima T: Derivation of human trophoblast stem cells. Cell Stem Cell 22: 50-63, 2018.

12. Hendrix MJ, Seftor EA, Hess AR and Seftor RE: Vasculogenic mimicry and tumour-cell plasticity: Lessons from melanoma. Nat Rev Cancer 3: 411-421, 2003.

13. Red-Horse K, Zhou Y, Genhacev O, Prakobphol A, Foulk R, McMaster M and Fisher SJ: Trophoblast differentiation during embryo implantation and formation of the maternal-fetal interface. J Clin Invest 114: 744-754, 2004.
14. Shih IeM: Trophoblastic vasculogenic mimicry in gestational choriocarcinoma. Mod Pathol 24: 646-652, 2011.

15. Keryer G, Alsat E, Tasken K and Evain-Brion D: Cyclic AMP-dependent protein kinases and human trophoblast cell differentiation in vitro. J Cell Sci 111: 995-1004, 1998.

16. Yurugi-Kobayashi T, Itoh $\mathrm{H}$, Schroeder T, Nakano A, Narazaki G, Kita F, Yanagi K, Hiraoka-Kanie M, Inoue E, Ara T, et al: Adrenomedullin/cyclic AMP pathway induces Notch activation and differentiation of arterial endothelial cells from vascular progenitors. Arterioscler Thromb Vasc Biol 26: 1977-1984, 2006.

17. Yamamoto T, Matsumoto K, Kurachi H, Okamoto Y, Nishio Y, Sakata M, Tasaka K and Murata Y: Progesterone inhibits transcriptional activation of human chorionic gonadotropin-alpha gene through protein kinase A pathway in trophoblast cells. Mol Cell Endocrinol 182: 215-224, 2001.

18. Belkacemi L, Beall MH, Magee TR, Pourtemour M and Ross MG: AQP1 gene expression is upregulated by arginine vasopressin and cyclic AMP agonists in trophoblast cells. Life Sci 82: 1272-1280, 2008.

19. Staun-Ram E, Goldman S and Shalev E: Ets-2 and p53 mediate cAMP-induced MMP-2 expression, activity and trophoblast invasion. Reprod Biol Endocrinol 7: 135, 2009.

20. Lee JM, Dedhar S, Kalluri R and Thompson EW: The epithelial-mesenchymal transition: New insights in signaling, development, and disease. J Cell Biol 172: 973-981, 2006.

21. Kalluri R and Weinberg RA: The basics of epithelial-mesenchymal transition. J Clin Invest 119: 1420-1428, 2009.

22. Sun T, Zhao N, Zhao XL, Gu Q, Zhang SW, Chen N, Wang XH, Du J, Liu YX and Sun BC: Expression and functional significance of Twist1 in hepatocellular carcinoma: Its role in vasculogenic mimicry. Hepatology 51: 545-556, 2010.

23. Liu Z, Sun B, Qi L, Li H, Gao J and Leng X: Zinc finger E-box binding homeobox 1 promotes vasculogenic mimicry in colorectal cancer through induction of epithelial-to-mesenchymal transition. Cancer Sci 103: 813-820, 2012.

24. Shawber CJ and Kitajewski J: Notch function in the vasculature: Insights from zebrafish, mouse and man. Bioessays 26: 225-234, 2004.

25. Vartanian A, Gatsina G, Grigorieva I, Solomko E, Dombrovsky V, Baryshnikov A and Stepanova E: The involvement of Notch signaling in melanoma vasculogenic mimicry. Clin Exp Med 13: 201-209, 2013.

26. Tian Q, Xue Y, Zheng W, Sun R, Ji W, Wang X and An R: Overexpression of hypoxia-inducible factor $1 \alpha$ induces migration and invasion through Notch signaling. Int J Oncol 47: 728-738, 2015.

27. Tang J, Wang J, Fan L, Li X, Liu N, Luo W, Wang J, Wang Y and Wang Y: cRGD inhibits vasculogenic mimicry formation by down-regulating uPA expression and reducing EMT in ovarian cancer. Oncotarget 7: 24050-24062, 2016.

28. Livak KJ and Schmittgen TD: Analysis of relative gene expression data using real-time quantitative PCR and the 2(-Delta Delta C(T)) method. Methods 25: 402-408, 2001.

29. Grummer R, Donner A and Winterhager E: Characteristic growth of human choriocarcinoma xenografts in nude mice. Placenta 20: 547-553, 1999.

30. Follin-Arbelet V, Hofgaard PO, Hauglin H, Naderi S, Sundan A, Blomhoff R, Bogen B and Blomhoff HK: Cyclic AMP induces apoptosis in multiple myeloma cells and inhibits tumor development in a mouse myeloma model. BMC Cancer 11: 301, 2011.

31. Hess AR, Seftor EA, Gruman LM, Kinch MS, Seftor RE and Hendrix MJ: VE-cadherin regulates EphA2 in aggressive melanoma cells through a novel signaling pathway: Implications for vasculogenic mimicry. Cancer Biol Ther 5: 228-233, 2006.

32. Paulis YW, Soetekouw PM, Verheul HM, Tjan-Heijnen VC and Griffioen AW: Signalling pathways in vasculogenic mimicry. Biochim Biophys Acta 1806: 18-28, 2010.

33. Cross JC,Hemberger M, Lu Y,Nozaki T, Whiteley K, Masutani M and Adamson SL: Trophoblast functions, angiogenesis and remodeling of the maternal vasculature in the placenta. Mol Cell Endocrinol 187: 207-212, 2002.

34. Kojima T, Katsumi A, Yamazaki T, Muramatsu T, Nagasaka T, Ohsumi K and Saito H: Human ryudocan from endothelium-like cells binds basic fibroblast growth factor, midkine, and tissue factor pathway inhibitor. J Biol Chem 271: 5914-5920, 1996.

35. Beese M, Wyss K, Haubitz M and Kirsch T: Effect of cAMP derivates on assembly and maintenance of tight junctions in human umbilical vein endothelial cells. BMC Cell Biol 11: 68, 2010.

36. Staun-Ram E and Shalev E: Human trophoblast function during the implantation process. Reprod Biol Endocrinol 3: 56, 2005. 
37. Langbein M, Strick R, Strissel PL, Vogt N, Parsch H, Beckmann MW and Schild RL: Impaired cytotrophoblast cell-cell fusion is associated with reduced Syncytin and increased apoptosis in patients with placental dysfunction. Mol Reprod Dev 75: 175-183, 2008.

38. Boize PA, Patrier S, Cheynet V, Oriol G, Massardier J, Hajri T, Guillotte M, Bossus M, Sanlaville D, Golfier F and Mallet F: Expression patterns of ERVWE1/Syncytin-1 and other placentally expressed human endogenous retroviruses along the malignant transformation process of hydatidiform moles. Placenta 39: 116-124, 2016.

39. Staun-Ram E, Goldman S, Gabarin D and Shalev E: Expression and importance of matrix metalloproteinase 2 and 9 (MMP-2 and -9) in human trophoblast invasion. Reprod Biol Endocrinol 2: 59, 2004.

40. Cohen M, Ribaux P, Epiney M and Irion O: Expression of metalloproteinases $1,2,7,9$, and 12 in human cytotrophoblastic cells from normal and preeclamptic placentas. Neuro Endocrinol Lett 33: 406-411, 2012

41. Bischof P, Martelli M, Campana A, Itoh Y, Ogata Y and Nagase H: Importance of matrix metalloproteinases in human trophoblast invasion. Early Pregnancy 1: 263-269, 1995.

42. Kessenbrock K, Plaks V and Werb Z: Matrix metalloproteinases: Regulators of the tumor microenvironment. Cell 141: 52-67, 2010

43. Li L and Li W: Epithelial-mesenchymal transition in human cancer: Comprehensive reprogramming of metabolism, epigenetics, and differentiation. Pharmacol Ther 150: 33-46, 2015.
44. Fan YL, Zheng M, Tang YL and Liang XH: A new perspective of vasculogenic mimicry: EMT and cancer stem cells (Review). Oncol Lett 6: 1174-1180, 2013.

45. Cuman C, Menkhorst E, Winship A, Van Sinderen M, Osianlis T, Rombauts LJ and Dimitriadis E: Fetal-maternal communication: The role of Notch signalling in embryo implantation. Reproduction 147: R75-R86, 2014.

46. Haider S, Pollheimer J and Knofler M: Notch signaling in placental development and gestational disease. Placenta 56: 65-72, 2017.

47. Huppert SS, Le A, Schroeter EH, Mumm JS, Saxena MT, Milner LA and Kopan R: Embryonic lethality in mice homozygous for a processing-deficient allele of Notch1. Nature 405: 966-970, 2000

48. Jasinska A, Strakova Z, Szmidt M and Fazleabas AT: Human chorionic gonadotropin and decidualization in vitro inhibits cytochalasin-D-induced apoptosis in cultured endometrial stromal fibroblasts. Endocrinology 147: 4112-4121, 2006. International (CC BY-NC-ND 4.0) License. 